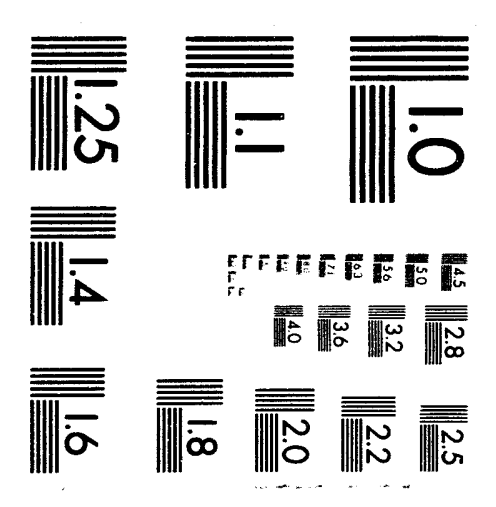



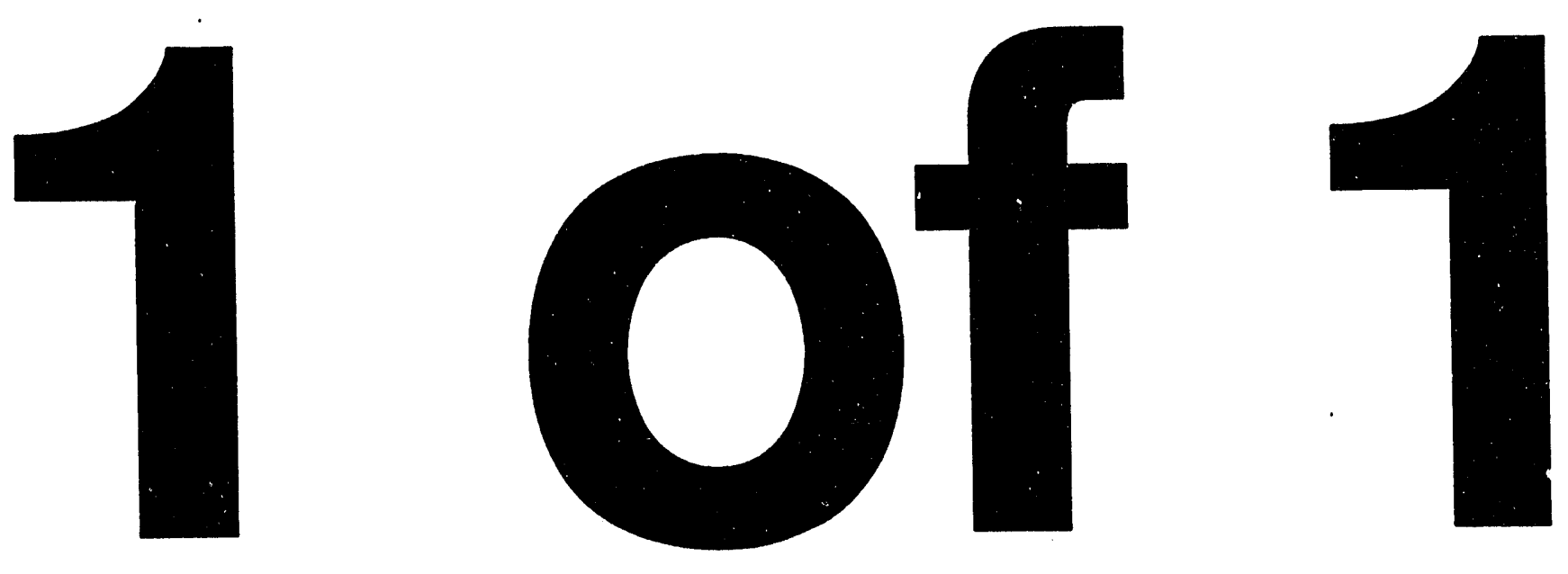
Survey Techniques Developed to Align Stacked Beamlines at CEBAF

C. J. Curtis, W. Oren, and K. J. Tremblay

Continuous Electron Beam Accelerator Facility

12000 Jefferson Avenue

Newport News, VA 23606

\section{Continuous}

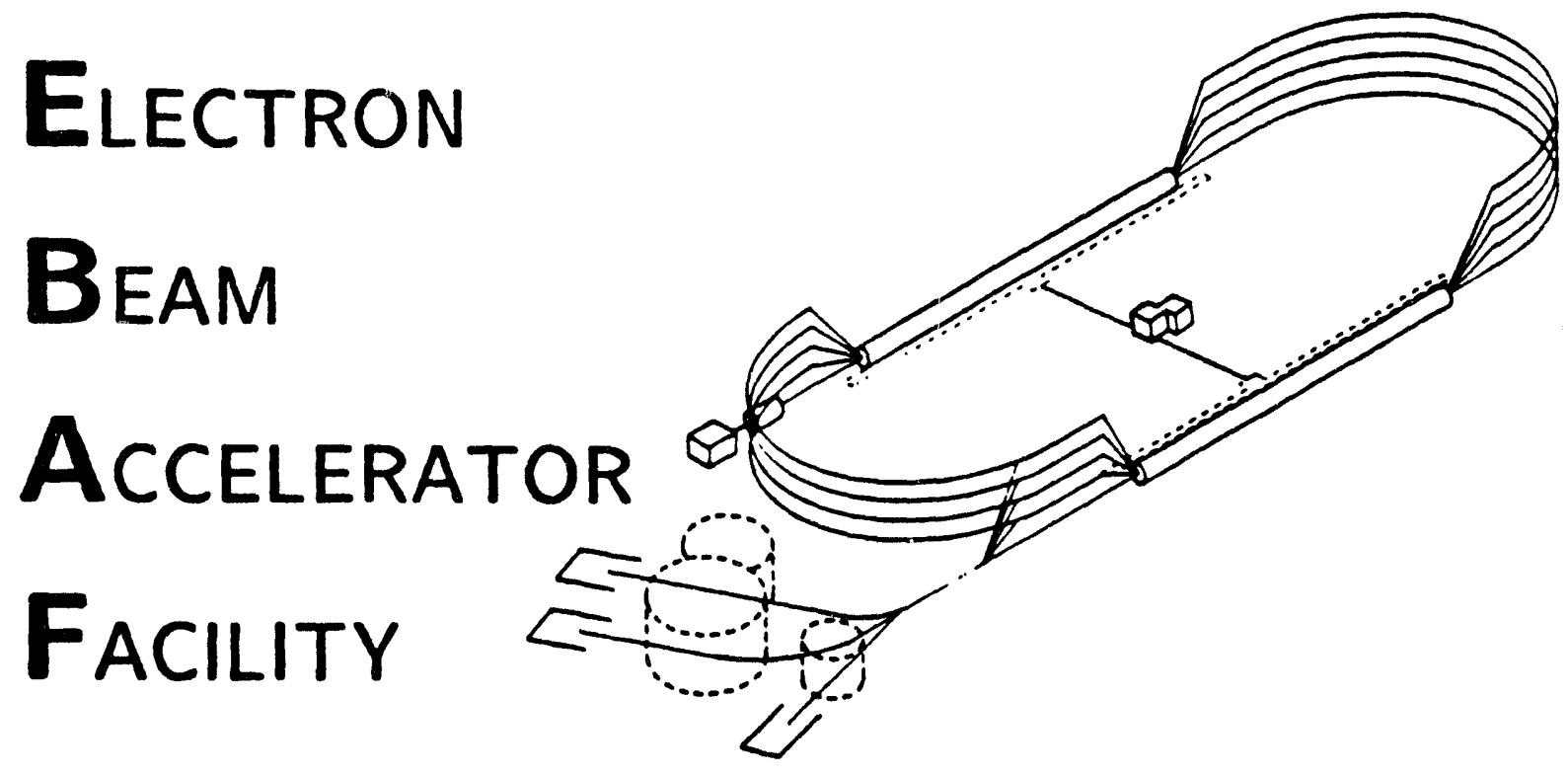

SURA SOUTHEASTERN UNIVERSITIES RESEARCH ASSOCIATION

CEBAF

Newport News, Virginia 


\title{
SURVEY TECHNIQUES DEVELOPED TO ALIGN STACKED BEAMLINES AT CEBAF*
}

\author{
C.J. Curtis, W. Oren, K.J. Tremblay \\ CEBAF, Newport News, Virginia, USA
}

\section{INTRODUCTION}

The Continuous Electron Beam Accelerator Facility (CEBAF) in Newport News, Virginia is a new accelerator designed to produce $4 \mathrm{GeV} 200$ micro-amp continuous wave beams for nuclear physics research. It consists of two superconducting linacs each accelerating electrons by $400 \mathrm{MeV}$ and linked by arcs allowing five pass recirculation. These linacs form the straight sections in a racetrack shaped accelerator contained in over $1.3 \mathrm{~km}$ of tunnel. The beam lines will consist of 42 superconducting accelerating cryomodules (in the linacs only), over 400 dipoles, 650 quadrupoles, and 100 sextupoles, most of the which are concentrated in the two arc sections of the machine. It is here that the single beam line from the linacs is split into five beams of differing energy and transported to the opposite linac where it is recombined into a single beam to again pass through a linac and receive additional acceleration.

These recirculation arcs are designed to maintain beam quality through a lattice which is achromatic, isochronous and whose length is equal to a multiple number of RF wavelengths [1]. The short term relative alignment tolerances coupled with the beam line design reflect the beam quality issues while absolute positioning determines the range of adjustment needed to match the RF phase in the linac segments. The alignment techniques which use a monumented control network as a reference, are designed to position stacked magnets and their suppon systems to these tolerances. Specialized procedures were tailored from existing hardware and software systems to address each phase or step of the alignment process. This allowed a relatively rapid expansion of alignment services at a new laboratory where surveying support was not seriously addressed until more then one third of the enclosure had been built.

\section{TOLERANCES}

In order to transport electrons around the arcs and deliver beam of acceptable quality to the succeeding linac, the alignment tolerances of magnets in the arcs have been defined as follows:

\footnotetext{
- Supported by U.S. DOE contract DE-AC05-84ER40150
} 
- relative position over $50 \mathrm{~m}$ transverse to the beam is $0.20 \mathrm{~mm}$ for quadrupoles,

$0.67 \mathrm{~mm}$ for sextupoles, and $0.50 \mathrm{~mm}$ for dipoles,

- absolute position for individual recirculation arcs is within $5.0 \mathrm{~mm}$ of ideal,

- linearity of the linacs should be within $2.0 \mathrm{~mm}$ of a straight line

The tolerance for quad positioning is a final design tolerance, with an initial alignment of $0.5 \mathrm{~mm}$ over $50 \mathrm{~m}$ required for the commissioning of the machine. All tolerances assume a Gaussian distribution truncated at $+/-2$ sigma for simulation.

\section{CONTROL NETWORK}

\subsection{Design}

The first stage in achieving the final tolerance was to establish a comprehensive control network. It was determined that this network would consist of stations in a zig-zag pattern around the accelerator such that those nearest to the beam line would permit the use of optical tooling techniques, and those furthest away would be more suited to theodolite measurements. This pattern also ensured that the majority of usable tunnel space was employed in order to strengthen the geometry of the traverse.

Pre-analysis using software developed at SLAC [2] was employed in order to determine an optimal observation scheme for the network which would be measured in two phases. In the first phase a "skeleton" network of almost 70 stations was measured which encircled the entire accelerator. These stations were to be of the highest order with error ellipses of less than $1.0 \mathrm{~mm}$. This scheme ensured a homogeneous and closed frame from which a densified network, necessary for the day to day operations, could be established when required.

\subsection{Survey Method and Equipment}

Survey monuments consist of stainless steel cups drilled and grouted into the tunnel floor. Into these, a 1.5 inch diameter half-sphere is placed to serve as a target either for directions or for centering a tripod. A full sphere provides a reference for levels. The SLAC system of fixed aluminum tripods and CERN sockets [3] was adopted because of its availability and the need to begin measurements as soon as the survey group arrived.

Direction observations were carried out using either the Kern E2 or the Wild T3000, and distances were measured with the Kern ME 5000. Leveling was carried out using a Wild N2 and double scale invar staves. Data was booked electronically onto the HP1 10 computer and transferred to PC's for reduction and adjustment using GEONET software [4]. 


\subsection{Results}

\subsubsection{First Skeleton Network}

Work first started on this scheme in September to October 1990. Orientation for the network was initially provided by the two main monuments utilized by the contractor in the civil construction. Transfer from these to the tunnel was carried out via two penetrations (see Fig 1) located in the north linac and extended to a network of 67 stations in the tunnel itself. The elevation datum was set by choosing the high point on the tunnel floor and defining it as the nominal distance below beam height.

The first skeleton network took approximately three weeks to measure. A minimally constrained adjustment was used with one of the north linac penetration points fixed in $\mathrm{Z}$ and $\mathrm{X}$, and the other fixed only in $\mathrm{X}$. The results largely followed the simulation with error ellipses of $0.4 \mathrm{~mm}$ along the north linac, and up to $0.7 \mathrm{~mm}$ along the south linac.

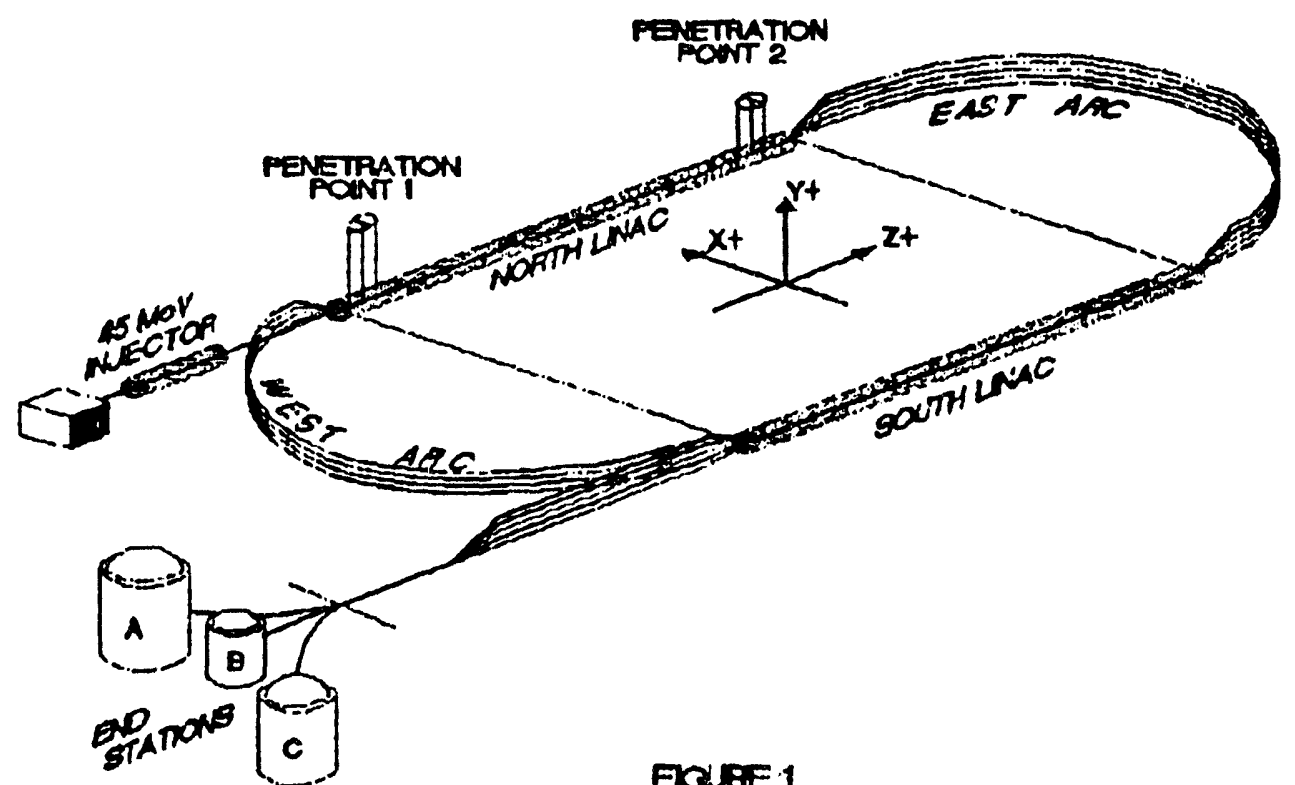

FOUFE 1

\subsubsection{Densification}

Once the skeleton network had been satisfactorily established it was possible to extend control to a densified scheme. This was first carried out in February 1991 in the north linac, east arc and part of the south linac, and added approximately 160 stations to the established points.

Initially the skeleton points were re-leveled and adjusted. Once it was determined that no significant vertical movement had taken place, the densified points were leveled 
and added to the adjustment. All of the horizontal network was observed concurrently (skeleton and densified), but again the skeleton points were adjusted first. Using a free net adjustment based on their previously defined positions, it was determined again that no significant movements could be detected. The positions defined by this free net adjustment were then accepted and used as fixed positions in a constrained adjustment of the densified points.

The densification work was carried out irregularly over a three month period. The full adjustment which held all skeleton points fixed produced error ellipses of less than $0.2 \mathrm{~mm}$ for the densified points.

The remaining densification for the accelerator was later completed in August 1991 when the injector, west arc and remaining part of the south linac were remeasured. Dut to local movements in the southwest comer of the accelerator, this portion of the densified network was oriented by fixing only the skeleton points in the northwest sector resulting in an acceptable adjustment.

\subsubsection{Maintenance}

Since the full accelerator was initially surveyed, the skeleton network has been remeasured twice. The first time was in April 1991 which served as a basis for the first densification scheme as described above.

The second time was in January 1992 and served as a basis for a full scale update of the accelerator control coordinates. The new skeleton observations were adjusted as a siee net and then subjected to a deformation analysis with the previous (April '91) data. This highlighted a maximum movement of over $2 \mathrm{~mm}$ in the north and east part of the accelerator. After optimization (i.e. minimizing movements in areas of installed components) a new set of coordinates for the skeleton network was obtained.

Time constraints had made it impossible to carry out a combined remeasurement of the skeleton and densified networks which now numbered over 300 points. For this reason, the possibility of using the updated skeleton network along with archived data for densified points was examined. The information from the most recent densification surveys for the entire accelerator was combined with the skeleton observations. Examination of the residuals from this adjustment highlighted areas where the old data was inconsistent with the new. In contrast to the deformation analysis, these areas were located in the north and west of the accelerator. The difference might be explained by a smooth movement which was indicated in the deformation analysis as opposed to a more irregular change indicated in the analysis of residuals. A densification survey was then carried out in these areas as well as in a small area which did not appear to have too many inconsistencies. The archived data was then replaced with the new observations in the final adjustment. The results were compared to the adjustment using only archived data and indicated coordinate changes of up to three times larger in the problem areas as compared to the non-problem areas. 
Although this method is not ideal, given the time constraints, it shuuld prove to be an adequate way of updating the network provided that an assumption of smooth movements is bome out in the field.

\section{STEP 1 ALIGNMENT}

\subsection{Concepts}

The Step 1 phase of alignment at CEBAF was designed to position support systems containing up to five levels of magnets. The typical arc stand consisted of up to ten mounting points machined so that their internal geometry was correct to $+1-2 \mathrm{~mm}$. Previous experience at SLAC [5] indicated that a point in space could easily be positioned to $+1-3 \mathrm{~mm}$ of its ideal three dimensional location as determined by intersecting lasers. This method would, in the worst case, leave $5 \mathrm{~mm}$ of the $+1-10 \mathrm{~mm}$ adjustment range available for positioning the magnet. For this reason, a system based on the CLASH program was developed.

While CLASH had proven effective for positioning a single pedestal where roll and pitch could be set with inclinometers, it did not directly lend itself to stands containing multiple levels of magnets. The construction of these stands prevented the control of their orientation angles with inclinometers and necessitated the simultaneous positioning of three registration points on the structure to control all six degrees of freedom. The other seven points then fell into place based on machining tolerances. To address this requirement, CEBASH was written based on a modified CLASH, providing for repeated pointings on consecutive targets so that stands could be iteratively moved to their correct positions.

\subsection{Cebash / Recebash}

The initial Step 1 alignment procedure utilized two theodolites centered over control points with vertical and horizontal orientation from backsights on adjacent monuments. As time progressed, the need for more flexibility in theodolite positioning and a desire to increase efficiency and accuracy lead to the development of RECEBASH. This program allowed for random setups of the theodolite pair whose locations were then solved for by resection.

RECEBASH requires the entry of the approximate tunnel location of the days work which allows the retrieval of both the ideal target coordinates and current horizontal and vertical control information. The program locates the target coordinates in a file of 3000 points and then uses this information to find the three dimensional coordinates of the adjacent control monuments. A minimum of five stations are required for the resections providing checks and ensuring a geometrically strong solution. After establishing the theodolite positions, three stand registration points which span the structure are observed to obtain its current orientation. Movements in the local beam following coordinate 
'system are output to the computer screen and ideal vertical and horizontal angles are displayed on the E2's. The stand is then iteratively moved to its ideal position as defined by the three registration points. A final observation on these points as well as the remaining registration points is made to record and check the results.

Due to manufacturing errors, some stands could not be positioned with all reference points within the $+1-3 \mathrm{~mm}$ alignment goal. In these cases, the procedure would be to average the differences and put the structure in its "best" location. Registration points $4 \mathrm{~mm}$ or more away from their ideal position were designated for mechanical fixes to be applied at the time of magnet installation. Further software development to incorporate as-built information for the stands geometry is needed to eliminate the time consuming process of splitting the difference in the field. As before the final location information is saved on file to complete the construction records.

\subsection{Results}

Soon after step 1 alignment was started an independent check was carried out to determine its effectiveness. In the check survey three rounds of angles were observed to determine the horizontal location of each cartridge, and precise leveling determined the elevation. The results of this survey were then compared with the as-found data generated in the CEBASH program. These proved to be remarkably good and are summarized in Table 1.

Table 1

Step 1 Alignment Verification (in mm)

\begin{tabular}{|c|c|c|c|}
\hline & DZ & DX & DY \\
\hline MEAN & 0.09 & 0.05 & 0.12 \\
\hline STD. DEV. & 0.12 & 0.07 & 0.07 \\
\hline
\end{tabular}

Typically seven to eight arc stands were aligned by a two to three man crew each day in the east arc, whereas this number rose to nine or ten stands per day in the west arc. The Step 1 procedure developed at CEBAF has proven to be a flexible and efficient method of aligning multi-beam stands.

\section{STEP 2 ALIGNMENT}

\subsection{Concepts}

The purpose of this procedure was to determine the position of magnets through a redundant observation plan reduced in a least squares adjustment. The differences between the actual magnet fiducial coordinates and their ideals could then be found and applied through adjustment systems monitored with electronic dial gages to move the magnet to its ideal location. 
Two survey methods were considered for achieving this goal. The first involved the traditional division of horizontal and vertical surveys with off line data analysis to calculate the necessary magnet motions. Although simulations provided acceptable results, the process was considered too time consuming and rather cumbersome. The second method, based on the three dimensional bundling systems commonly used in industry, seemed to fit the problem nicely. The stacked beam lines provided a strong model space both in the vertical and horizontal dimensions while todays powerful compact computers furmished the capacity for large customized programs. With some help from SLAC, the Stanford Industrial Measurement System (SIMS) [6] was adapted to fit CEBAFs needs. SIMS was nested within an operating shell of CEBAF design which stepped the user through a customized observation and data analysis routine.

\subsection{Design}

As was previously mentioned, the control network was designed to accommodate both optical tooling or theodolite based methods for magnet placement. Simulations and actual test measurements proved that the zig-zag pattern of monuments provided a strong basis (sigmas of 60 microns or less) for a bundling measurement of the five levels of magnets. The beam line was then partitioned into logical units (sectors) of 7.5 degrees with up to 25 magnets, to fit a measurement space spanned by three theodolites. Within this model, the instruments could be positioned within one meter of their simulated location to achieve satisfactory geometry and the desired error envelopes.

Each magnet was fitted with a fixture containing two fiducial marks similar to the floor monuments. The fixtures registered on reference surfaces machined into the magnet at the time of manufacture. As is commonly done, these surfaces were assumed to represent the magnetic centerline and therefore the actual beam line. Each magnet was dimensionally inspected upon arrival at CEBAF for conformance to design. Similarly, the 80 fixtures of three different designs were inspected on a coordinate measuring machine to obtain precise offsets for each target which were then factored into the ideal coordinate calculations.

\subsection{Software}

With the locations of the magnet references defined and the design of an observation scheme set, the intention was to create a robust software system to control the complete process of surveying and aligning the magnets in the field. To make the package as friendly as possible, it was assumed that the user would be inexperienced in adjustment procedures, computers and modem accelerator alignment techniques. Additionally, checking routines to look for common errors such as improper element or control point names as well as pointing errors, were included. This led to the development of the Step 2 Arc Alignment system (S2AA).

The program starts by asking the user for a single magnet name in the sector of interest. This leads to the creation of a series of menus and lists where the operator 
selects the necessary magnet and control point names and designates fixture locations and serial numbers. This information is combined with current fixture calibration reports and DIMAD [7] beam line layout data to produce ideal target coordinates for use throughout the program. To assure that current data is utilized, a system of master disks controlled by the data manager was setup. These disks are updated by the data manager and must be present to initialize the program.

After the measurement campaign has been set up, a series of files are created to record the results and ensure that previous data is not over-written. The crew then collects the observations using the CAPTURE routine from SIMS. Upon completion of the measurements, a check of the observed angles is made by comparing them to ideal values derived from preliminary estimates of the theodolites positions. If the field data does not match an angular tolerance based on the distance from the target to the theodolite, the database is searched to see if the observation might match a different target. If a match is found, the operator is given the choice of swapping the target names. If no match is found, the data can be rejected and processing continued. A final manipulation of the horizontal angles then takes place so that the bundle adjustment can be carried out in the overall machine coordinate system.

The SIMS bundling routine is then used to obtain actual target coordinates which are compared to their ideals. Based on these results and the geometric configuration of the adjustment systems, S2AA calculates the necessary dial gage motions to move the magnet to its proper position. A printout is produced to guide the adjustments and provide a paper record of the final dial gage readings.

A second survey is then performed to check the results. At this point the fixtures are shuffled to remove placement errors which could have occurred in the first survey. If one or two of the magnets are then found to be out of tolerance they are moved and reobserved. S2AA provides a method where the reobservations of these magnets can then be "patched" into the raw observation file to eliminate the reobservation of undisturbed magnets and maintain the setups robust geometry. Finally, a subset of data files and results are transferred to the office for archiving and progress tracking.

\subsection{Results}

Three tests have been carried out which indicate the type of accuracy achievable with the S2AA system. The first of these involved the measurement of five quadrupoles and four sextupoles with both the S2AA method and optical tooling. On each magnet two targets were observed. The average differences in the $X$ and $Y$ coordinates were $0.060 \mathrm{~mm}$ with a standard deviations of $0.040 \mathrm{~mm}$ or less. Insufficient data was available for the $\mathrm{Z}$ coordinate.

The second test was carried out in order to verify the repeatability of the target fixtures. In this test a single S2AA setup was made on a sector consisting of nine quadrupoles. After one set of measurements had been taken, the fixtures were removed 
and replaced on the adjacent magnet such that another set of measurements could be made. In this way the fixtures were rotated around the stacks up to five times. As such this test gives an indication of the accuracy of only part of the survey procedure. In general, the repeatability of the fixtures in $X$ and $Y$ was better than in $Z$. The standard deviation for $X$ and $Y$ repeatability was of the order of $0.020 \mathrm{~mm}$ whereas for $Z$ it was $0.040 \mathrm{~mm}$.

The third test involved a complete remeasurement of seven sectors of magnets. The measurements took place approximately two months apart and utilized the S2AA method. This test gives a good indication of the repeatability of the system as a whole. The mean of the absolute differences for upstream and downstrean $Z, X$, and $Y$ targets for all seven sectors are summarized in Table 2. These results again indicate a precision in $Z$ of roughly half that in $X$ and $Y$. This is neither surprising given the design of the fixtures and the survey system, nor of any concern given the much lower alignment tolerances in the $\mathrm{Z}$ direction $(5 \mathrm{~mm})$. The overall magnitude of the differences is very satisfying when judged against the desired survey precision.

Table 2

Repeatability of S2AA Surveys (in mm)

\begin{tabular}{|c|c|c|c|c|c|c|}
\hline & $\mathbf{Z u}$ & $\mathbf{X u}$ & $\mathbf{Y u}$ & $\mathbf{Z d}$ & $\mathbf{X d}$ & $\mathbf{Y d}$ \\
\hline Mean & 0.071 & 0.040 & 0.035 & 0.089 & 0.046 & 0.042 \\
\hline Std Dev & 0.254 & 0.112 & 0.098 & 0.293 & 0.126 & 0.135 \\
\hline
\end{tabular}

The normal rate of production for a three man crew is approximately one sector per shift. This met our original time estimates for Step 2 alignment in the arcs. This software package has been successfully adapted for use in other areas of the accelerator and further improvements are envisioned to expand its use throughout the machine.

\section{CONCLUSION}

Tests of the beam line up to the 135 degree point of the east arc partially prove the validity of the alignment methods discussed in this paper. Over 350 multiple level stands have been successfully aligned with mechanical fabrication errors identified for remedial action. Almost all of the primary magnetic elements have been aligned to the commissioning tolerance of $+1-0.50 \mathrm{~mm}$ over 50 meters, with the east arc and north linac test proving that the components are well within this error band. While the methods described here are not revolutionary in nature, they do prove that careful application of current technology can produce a successful alignment of a large multi-pass electron accelerator for nuclear physics. 


\section{REFERENCES}

[1] CEBAF Design Report, May 1986.

[2] I. Burstedde, "Das Programmsystem GEONET zur Ausgleichung gedatischer Netze," in: Allgemeiner Vermessungs-Nachrichten, 3, 1987, pp. 118-130.

[3] M. Pietryka, H. Friedsam, W. Oren, R. Pitthan and R. Ruland, Proc. of ASPACSM Spring Meeting, 1985, p.321

[4] R. Pushor, Geonet Data Management System Users Guide, unpublished SLAC documentation, 1987.

[5] C.J. Curtis, W. Oren, and R. Ruland, Proc. of ASP-ACSM Spring Meeting, 1986, Vol 2, p.61.

[6] J. M. Gaunt, "Bundle Adjustments and Tridimensional Coordinate Determination", SLAC-PUB-4714, 1988.

[7] R.V. Servranckx, et. al., "Users Guide to the Program DIMAD", SLAC Report 285 UC-28 May 1985. 

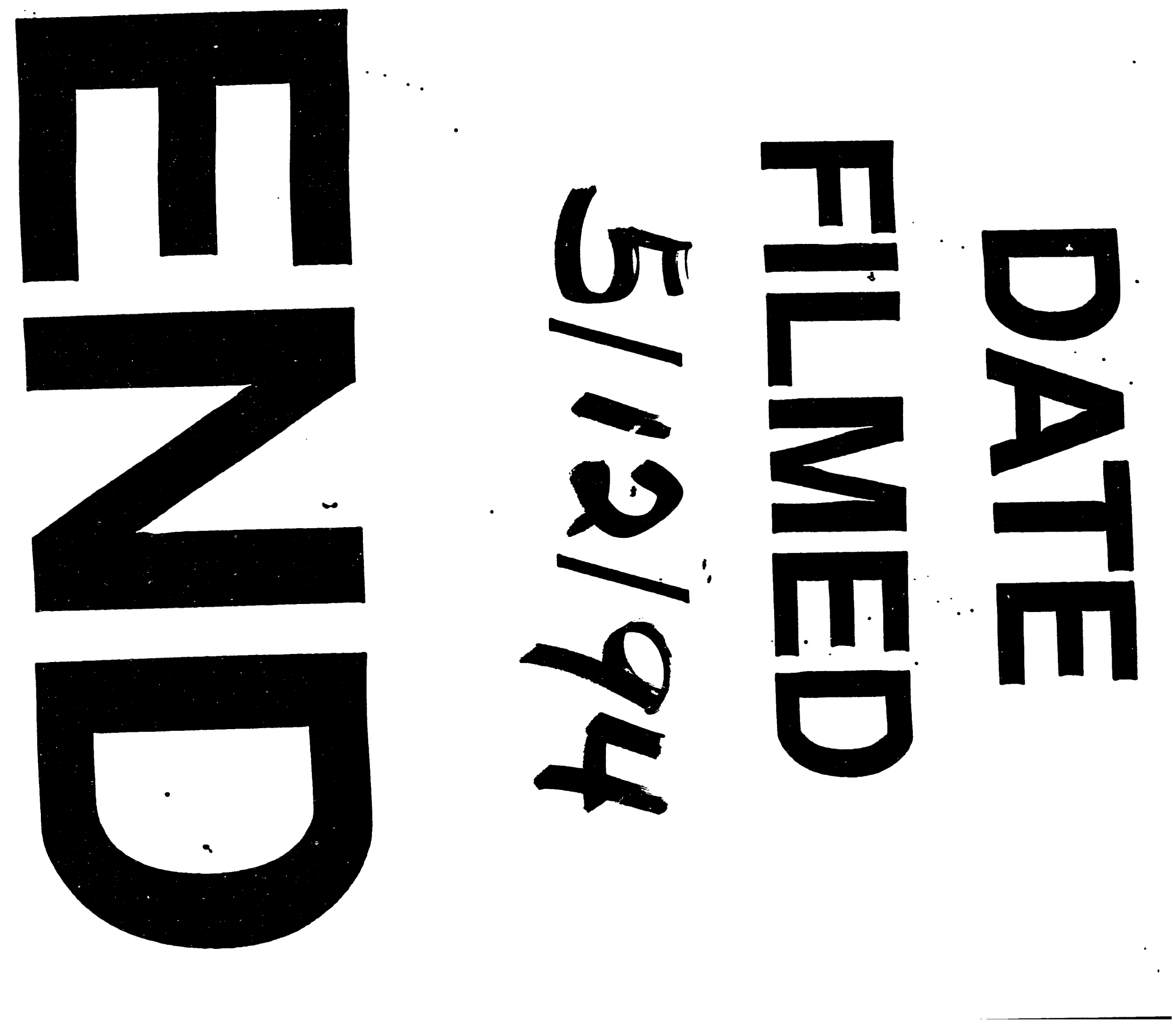
